\title{
Meißner, Florian: Kulturen der Katastrophenberichterstattung. Eine Interviewstudie zur Fukushima-Krise in deutschen und japanischen Medien
}

\author{
Wiesbaden: Springer VS 2019. 440 Seiten. Preis: €39,99 (e-book)
}

\author{
Alexander Görke \\ Online publiziert: 11 . März 2020 \\ (C) Der/die Autor(en) 2020
}

Krisen entstellen den Normalverlauf von Systemen bis zur Kenntlichkeit, so hat Siegfried Weischenberg einmal die Wirkweise von Krisen auf die betroffenen Systeme beschrieben und damit zugleich auch verdeutlicht, warum speziell die Analyse von Krisen für Kommunikationswissenschaftler interessant ist. In der vorliegenden Studie, die als Dissertation an der TU Dortmund eingereicht wurde, widmet sich Florian Meißner einem Krisenfall, der kaum zu toppen ist: der Analyse der DreifachKatastrophe von Fukushima und ihrer Aufarbeitung durch deutsche und japanische Journalisten.

Gleich zu Beginn der Studie benennt Florian Meißner drei zentrale Gütekriterien internationaler Journalismusforschung, die indes längst nicht von allen Ansätzen der komparativen Journalismusforschung geteilt werden. Hierzu zählt der Autor neben der Mehrebenen-Systematik auch die Äquivalenz der untersuchten Phänomene und vor allem die Forderung nach einer De-Westernization der Journalismusforschung und ihrer Theorien und Modelle. Um eine Gleichsetzung von „westlich“ und ,modern“ zu umgehen, schließt sich der Autor der Forderung an, die kulturellen Divergenzen und Diversitäten der nicht-westlichen Welt stärker zu berücksichtigen. Deshalb spricht der Autor dann auch davon, mit dem Forschungsparadigma „Journalismuskultur“ zu arbeiten, dem eine Reihe unterschiedlicher Konzeptansätze zuzuordnen seien, wobei auch eingeräumt wird, diese seien hinsichtlich ihrer Begriffsverständnisse notorisch unklar und zudem selbst kulturspezifisch. Um dennoch mit dem Paradigma „Journalismuskultur“ arbeiten zu können, ist die Suche nach einer Art (kleinstem) gemeinsamen Nenner erforderlich. Hierbei orientiert sich der Verfasser an den Vorarbeiten von Esser und Hanitzsch, die vor allem die Wech-

Prof. Dr. A. Görke $(\bowtie)$

Institut für Publizistik- und Kommunikationswissenschaft, Freie Universität Berlin,

Garystr. 55, 14195 Berlin, Deutschland

E-Mail: Alexander.Goerke@fu-berlin.de 
selbeziehung zwischen der journalistischen Kulturproduktion und ihrem jeweiligen kulturellen Kontext in den Blick nehmen. Im Folgekapitel werden dann nach dem Vorbild des „Hierarchy of Influences Model“ von Shoemaker and Reese (2014) verschiedene Einflussebenen in Bezug auf Journalismus in Deutschland und Japan benannt und einer mehrstufigen Analyse unterzogen. Neben der Ebene der sozialen Systeme und der sozialen Institutionen werden hierbei die Ebenen der Medienorganisationen, der journalistischen Routinen sowie der Individuen unterschieden und feingliedrig aufgeschlüsselt. Auf diese Weise generiert Meißner eine sehr kompakte Darstellung der Differenzen zwischen japanischem und deutschem Journalismus.

Das Folgekapitel führt dann in das Thema Katastrophenberichterstattung ein, liefert Begriffsklärungen und eine Schilderung der Dreifach-Katastrophe von Fukushima, wobei auch die Besonderheiten der deutschen und japanischen Berichterstattung angesprochen werden. Insbesondere erfährt der Leser hier auch etwas über die innerjapanischen Unterschiede zwischen nationalen und regionalen Medien einerseits und der Kluft zwischen traditionellen Medienanbietern und der Online-Kommunikation.

Die Studie setzt auf ein Mehrmethodendesign aus Expertenbefragung und narrativen Interviews. Zu Recht führt der Autor aus, dass aufgrund der vorliegenden inhaltsanalytischen Studien zum Thema ,die unterschiedlichen Charakteristiken der deutschen und japanischen Berichterstattung bereits recht genau beschrieben“ (S. 190) wurden. Meißner will hingegen mit seiner Rekonstruktion der Rahmenbedingungen, die als unterschiedliche Journalismuskulturen vorgestellt werden, einen Schritt weitergehen. Er hält es deshalb auch für ,,naheliegend, wenn nicht zwingend, für die vorliegende Arbeit einen theoretisch offenen, sprich induktiven Forschungsansatz zu wählen“ (S. 184). Beides kann man vor dem Hintergrund unterschiedlicher Theorieangebote in der Journalistik durchaus anders sehen. Denn in der Tat fördern die Befunde der Befragungen so einiges zu Tage, was sich mit dem Forschungsstand deckt. Deutlich wird, dass die deutschen Medien Fukushima vor allem vor dem Hintergrund der deutschen Atomenergiedebatte eingeordnet und bewertet haben. „In beiden Gesellschaften besitzt das liberale (angelsächsische) JournalismusModell eine Vorbildfunktion. Diese wird allerdings in Deutschland stärker realisiert als in Japan, wo endogene Berichterstattungstraditionen weiterhin eine große Rolle besitzen“ (S. 377). Hierzu zählt der Autor vor allem den Einfluss der Reporterclubs auf die Quellenauswahl, das letztlich zu Lasten der journalistischen Autonomie geht. Auch über die Kritik der japanischen Bevölkerung an dieser Form der Katastrophenberichterstattung kann man einiges erfahren, wie auch über die Versuche der traditionellen Medien, stärker investigativ zu arbeiten und sich aus dem Griff der staatlichen Interessen zu lösen. Meißner zeigt auf, dass diese Ausbruchsversuche nicht von langer Dauer gewesen sind, auch weil auf die etablierten Medien erheblicher politischer Druck ausgeübt wurde. Alles in allem ist das eine ernüchternde Bilanz, die die Frage aufwirft, ob hier die Apriori-Setzung einer kulturellen Imprägnierung des Journalismus und die Überzeugung, einen Beitrag zur De-Westernization von Journalismusmodellen erbringen zu müssen, nicht auch als Entschuldigungsargumente herhalten müssen. Diese Alternative sollten Leser und Leserinnen der Studie zumindest mitdenken, wenn Meißner den Stellenwert der eigenen Studie bilanziert: „Ein Großteil der international verfügbaren Werke zum japanischen Journalismus analysiert und kritisiert diesen vor allem nach Maßstäben 
des liberalen Journalismus-Modells angelsächsischer Prägung. Abweichungen von diesem idealisierten Modell wurden als Defizite oder, aus entwicklungstheoretischer Betrachtung, gar als Ausweis mangelnder Reife interpretiert. Die vorliegende Studie wählte einen völlig anderen Weg, dessen entscheidender Ausgangspunkt der kulturelle und historische Kontext journalistischer Aussageproduktion war" (S. 395). Wie wahr. Aber werden deshalb aus journalistischen Defiziten schon Journalismuskulturen?

Funding Open Access funding provided by Projekt DEAL.

Open Access Dieser Artikel wird unter der Creative Commons Namensnennung 4.0 International Lizenz veröffentlicht, welche die Nutzung, Vervielfältigung, Bearbeitung, Verbreitung und Wiedergabe in jeglichem Medium und Format erlaubt, sofern Sie den/die ursprünglichen Autor(en) und die Quelle ordnungsgemäß nennen, einen Link zur Creative Commons Lizenz beifügen und angeben, ob Änderungen vorgenommen wurden.

Die in diesem Artikel enthaltenen Bilder und sonstiges Drittmaterial unterliegen ebenfalls der genannten Creative Commons Lizenz, sofern sich aus der Abbildungslegende nichts anderes ergibt. Sofern das betreffende Material nicht unter der genannten Creative Commons Lizenz steht und die betreffende Handlung nicht nach gesetzlichen Vorschriften erlaubt ist, ist für die oben aufgeführten Weiterverwendungen des Materials die Einwilligung des jeweiligen Rechteinhabers einzuholen.

Weitere Details zur Lizenz entnehmen Sie bitte der Lizenzinformation auf http://creativecommons.org/ licenses/by/4.0/deed.de.

Dr. Alexander Görke ist Professor am Institut für Publizistik- und Kommunikationswissenschaft der Freien Universität Berlin mit den Schwerpunkten Wissenskommunikation und Wissenschaftsjournalismus. 